\title{
Cloves protect the heart, liver and lens of diabetic rats
}

\begin{abstract}
Clove oil was previously reported to modulate physiological responses in streptozotocininduced diabetic rats. This study attempts to evaluate the in vivo organ and tissue protective effects of dietary cloves (Eugenia aromaticum) in chronic hyperglycaemia. The cloves (equivalent to $100 \mathrm{mg}$ total eugenol + eugenyl acetate per $\mathrm{kg}$ body weight/day) were administered orally to streptozotocin-induced diabetic male Sprague-Dawley rats. Fasting blood glucose levels, as well as organ tissue physical and biochemical markers, were monitored. Dietary supplementation with cloves reduced tissue injuries, especially in the lens and cardiac muscles, and to a lesser extent in the liver but not the kidneys. Additionally, the cloves treatment significantly reduced blood sugar increases and lipid peroxidation in streptozotocin-induced diabetic rats by restoring the antioxidant enzyme levels. Cloves inhibited hyperglycaemia-induced oxidative tissue damage and cataract formation in the eye lens. This study demonstrates the in vivo antioxidative organ protective effects of clove in diabetics.
\end{abstract}

Keyword: Cloves; Eugenol; Myocardium; Eye lens; Kidney; Liver; Diabetes 